\title{
LA MEJORA DE LA CONVIVENCIA EN EL MARCO DE LA ESCUELA UNITARIA COMO PUENTE PARA EL APRENDIZAJE
}

The improvement of coexistence in the framework of the unitarian school as a bridge for learning

Melhorar a coexistência no âmbito da escola unitária como uma ponte para a aprendizagem

\section{Marina Gómez Rodríguez}

CRA El Pizarral, Santa María la Real de Nieva (Segovia). España. Teléfono: +34 636627584. Correo electrónico: marinagomezro@gmail.com

\section{Resumen}

La siguiente experiencia tiene como finalidad la mejora de la convivencia en una escuela unitaria, demostrando así la importancia que alberga la existencia de un buen clima de aula para el aprendizaje del alumnado. Este aspecto se trabaja en tres líneas fundamentales. La primera de ellas gira en torno al aprendizaje cooperativo, el cual constituye una herramienta muy útil para la creación un sentimiento de necesidad mutua y positiva entre los miembros del grupo, trabajando todos unidos para lograr el aprendizaje individual. En este sentido, el uso de la asamblea constituye un momento de comunicación y convivencia excelente, favoreciendo la adquisición de las normas más básicas de la sociedad democrática. El último aspecto trabajado son los procesos de coevaluación. Estos sin duda permiten que algunos de los niños comiencen a aceptar las opiniones y consejos de sus iguales, mientras que en otros logran aumentar su autoestima al sentirse valorados grupalmente.

Palabras clave: Convivencia; escuela unitaria; coevaluación; aprendizaje cooperativo; asamblea

\begin{abstract}
The aim of the following experience is the improvement in the coexistence in a unitarian school, proving the importance that a nice class environment has in the students' learning. This aspect is worked out through three basic lines. The first one goes around cooperative learning, which is a useful tool for the creation of a mutual and positive feeling of necessity between the members of the group; who work together to La mejora de la convivencia en el marco del aula unitaria como puente para el aprendizaje
\end{abstract}


achieve an individual learning. In this sense, the use of the assembly supposes an excellent moment to communicate and coexist, that favours the acquisition of democratic social basic rules. The last element referred to is the co-assessment processes. They, undoubtedly, allow to some children to start to accept the opinions and advice of their peers, and at the same time, some others get to enhance their self-esteem as they feel valued by the group.

Keywords: Coexistence; unitarian school; co-assessment; cooperative learning; assembly

\section{Resumo}

A experiência seguinte tem como finalidade a melhoria da convivência em uma escola unitária, demonstrando assim a importância que alberga a existência de um bom clima de aula para o aprendizado do aluno. Este aspecto é trabalho em três linhas fundamentales. A primeira de gira em torno do aprendizado cooperativo, o que é uma ferramenta muito útil para a criação de um sentimento de necessidade mútua e positiva entre os membros do grupo, todos os unidos para conseguir o aprendizado individual. Em este sentido, o uso da assembléia é um momento de comunicação e convivência excelente, favorecendo a aquisição das normas mais básicas da sociedade democrática. O último aspecto é trabalhado no processo de coevaliação. Estes sem dúvida permitem que alguns dos jovens adquiram as opiniões e os conselhos de seus amigos, enquanto que outros países aumentam sua autoestima e sentem-se valorizados grupalmente.

Palavras-chave: Coexistência; escola unitária; coavaliação; aprendizagem cooperativa; assembleia

\section{Introducción}

El ámbito de la escuela unitaria es el gran desconocido de la educación. Las características de la misma hacen de ella un contexto educativo que entraña multitud de dificultades, entre ellas, la de la convivencia.

Todo centro educativo, por muy pequeño que sea, constituye una parte de la sociedad y, por tanto, es reflejo de la misma. En ella los alumnos aprenden a relacionarse, a cooperar y en definitiva a vivir juntos, por lo que forma parte de los que Delors (1996) considera los cuatro pilares básicos de la educación. 
De ahí la importancia de aplicar los principios de la llamada escuela democrática como una "institución que se plantea instruir y formar a los alumnos propiciando su participación junto con la de los maestros en el trabajo y la convivencia escolar” (Escardíbul, Martín, Novella y Puig, 1999, p.17).

Así mismo, un adecuado clima de convivencia favorece el adecuado aprendizaje, no solo por mejorar el ámbito de trabajo, sino fundamentalmente por instaurarse una cultura de ayuda en el aula, principio que se ajusta perfectamente a los objetivos de aprendizaje cooperativo, que permite que los alumnos trabajen juntos para maximizar, no solo su propio aprendizaje sino también el de los demás (Johnson, Johnson y Holubec, 1999). El aprendizaje, por tanto, se encuentra "al servicio de una "comunidad" -de un equipo, de un grupo clase...” (Pujolás, 2002, p.7) lo cual inevitablemente provoca un sentimiento de pertenencia al grupo, mejorando las herramientas de convivencia y relación social de cada individuo.

\section{Contextualización}

Las propuestas detalladas a continuación se enmarcan en el colegio de la localidad de Codorniz, perteneciente al CRA “El Pizarral” con cabecera en Santa María la Real de Nieva (Segovia). Se trata de un aula unitaria con ocho alumnos incluidos en los niveles de tres y cuatro años de Educación Infantil, así como de segundo, tercero, cuarto y sexto de Educación Primaria.

\section{Diseño y desarrollo}

La siguiente propuesta comienza a principios del curso 2016/2017, partiendo de la realización de una evaluación inicial del grupo clase. Para ello, en primer lugar, se utilizó un sociograma, que permitió, junto con los datos de la observación diaria en el aula, comprender que existía un problema de convivencia entre el alumnado.

Por este motivo se decidió llevar a cabo varias propuestas encaminadas a la mejora de estos aspectos, que sin duda repercutían negativamente en el aprendizaje de los niños, al causar un mal clima de trabajo en el aula ausente de cooperación, ayuda y respeto.

En primer lugar, se comenzó con la aplicación de técnicas de aprendizaje cooperativo, tratando de favorecer con ello la llamada interdepencencia positiva (Johnson \& Johnson, 1999) entre los miembros del grupo. Al no haber trabajado 
previamente en el aula de forma cooperativa, se comenzó colocando al alumnado en parejas, así como estableciendo unos roles a nivel de aula, dada la complejidad de la misma. Para ello se tomaron muy en cuenta las propuestas de los niños, que decidieron darle una estructura semejante a la de un ayuntamiento, en el que cada alumno tiene un cargo durante un mes, en que tiene que desarrollar dos funciones diariamente en clase.

Entre las propuestas de aprendizaje cooperativo utilizadas destacan para Educación Primaria: “Gemelos lectores”, “Dictado en parejas” y "Lápices al centro”; y para Educación Infantil: "Entrevista simultánea” y colocación cooperativa de babis, todas ellas con muy buenos resultados.

En esta línea de trabajo para la mejora de la convivencia y con ella de los resultados de los alumnos, se introducen técnicas de coevaluación en el aula, consiguiendo, de este modo, una coherencia entre la práctica educativa y los planteamientos pedagógicos previos (López-Pastor, 2006). La coevaluación se utiliza en el aula a través de procesos sencillos como el uso de listas de control para aspectos relativos a exposiciones orales y a la realización de textos en el alumnado de Educación Primaria.

La tercera vía de trabajo para la mejora de la convivencia gira en torno a las asambleas semanales que toman como base el diálogo, iniciándose un proceso de prevención y resolución de conflictos en el que todas las personas participan, extendiéndose este a en todos los momentos de la jornada (Flecha y García-Yeste, 2007, p.72). En las asambleas, por un lado, se evalúa el desempeño de las funciones de cada uno de los miembros de grupo, tratando de que todo el alumnado se implique en ayudar a cada persona a desempeñar de forma más exitosa sus responsabilidades. Por otro lado, la asamblea constituye un momento de comunicación de sentimientos, ideas y emociones al grupo de forma libre, facilitándose de este modo el conocimiento mutuo y el respeto. Así mismo la asamblea proporciona un vehículo de aprendizaje a través del trabajo de temas como el acoso escolar o incluso las problemáticas surgidas en el aula, que facilitan la adquisición de conocimientos y herramientas de solución de conflictos y de convivencia social.

\section{Evaluación}

Estas propuestas, aunque sencillas, supusieron un punto de inflexión en el clima de la clase. El comienzo de curso resulto ciertamente dificultoso, en gran medida por la 
necesidad de asimilar los nuevos hábitos de trabajo. No obstante, la constancia consiguió dar sus frutos. El diálogo y el permitir al alumnado tener su propio papel y voz dentro del proceso de enseñanza-aprendizaje mejoró su implicación en el mismo.

Las propuestas explicadas y otras que poco a poco se han ido incluyendo en la rutina del grupo son sin lugar a dudas una parte fundamental de la actual cultura de cooperación existente en el aula, gracias a la cual se ha producido progresivamente una mejora sustancial en la convivencia y en definitiva en el aprendizaje del alumnado.

\section{Conclusiones}

Esta experiencia de trabajo en tres líneas para la mejora de la convivencia puede aplicarse a cualquier grupo clase independientemente del número de alumnos y curso. Las circunstancias más propicias para obtener óptimos resultados serían las de su uso coordinado a nivel de centro como una línea común de trabajo, al crearse con ello una cultura de cooperación y convivencia armoniosa.

\section{Referencias}

Delors, J. (1996.). Los cuatro pilares de la educación La educación encierra un tesoro. Informe a la UNESCO de la Comisión internacional sobre la educación para el siglo XXI (p. 91-103). Madrid: Santillana/UNESCO.

Escardíbul, S., Martín-García, X., Novella, A.M., \& Puig, J.M. (1999). Cómo fomentar la participación en la escuela. Propuestas de actividades. Barcelona: Graó.

García, R. F., \& Yeste, C. G. (2007). Prevención de conflictos en las comunidades de aprendizaje. Idea La Mancha: Revista de Educación de Castilla-La Mancha, (4), 72-76.

Recuperado

de http://cefire.edu.gva.es/pluginfile.php/329694/mod_resource/content/1/FlechaGar cia_Prevenci\%C3\%B3n\%20de\%20conflictos\%20en\%20las\%20comunidades.pdf

Johnson, D.W., Johnson, R.T., \& Holubec, E.J. (1999). El aprendizaje cooperativo en el aula. Buenos Aires: Paidós.

López-Pastor, V. (coord.) (2006). La evaluación en la Educación Física: revisión de los modelos tradicionales y planteamiento de una alternativa: la evaluación formativa y compartida. Buenos Aires: Editorial Miño y Dávila. 
Pujolás, P. (2002). El aprendizaje cooperativo. Algunas propuestas para organizar de forma cooperativa el aprendizaje en el aula. Documento de trabajo. Universidad de Vic: Laboratorio de psicopedagogía. Recuperado de http://www.deciencias.net/convivir/1.documentacion/D.cooperativo/AC_Propueta sorganizativas_Pujolas_39p.pdf. 